\title{
Performance Analysis of path tracking using Multi-Dimensional Kalman Filter based on state estimations
}

\author{
Praveenkumar Babu, Student Member, IEEE and Eswaran Parthasarathy
}

\begin{abstract}
In the presence of uncertainty, one of the most difficult issues for tracking in control systems is to estimate the accuracy and precision of hidden variables. Kalman filter is considered as the widely adapted estimation algorithm for tracking applications. However, tracking of multiple objects is still a challenging task to achieve better results for prediction and correction. To solve this problem, a multi-dimensional Kalman filter is proposed using state estimations for tracking multiple objects. This paper also presents the performance analysis of proposed tracking model for linear measurements. The steadystate and covariance equations are derived and their co-efficients are updated. The multi-dimensional Kalman filter is evaluated mathematically for linear dynamic systems. The path tracking based on Kalman filter and multi-dimensional Kalman filter is also analyzed. The true and filtered responses of our proposed filtering algorithm for multiple object tracking are observed. The output covariance produces steady state values after four number of samples. The simulation results shows that the performance of our proposed filtering algorithm is $2 x$ times effective than conventional Kalman filter for objects moving in linear motion and proves that proposed filter is suitable for realtime implementation.
\end{abstract}

Index Terms-Correction process, updated Covariance equation, Kalman filter, linear dynamic systems, multiple object tracking, prediction and steady-state equations.

\section{INTRODUCTION}

The accuracy of tracking moving objects is one of the key challenges in remote monitoring systems. Kalman filter $(\mathrm{KF})$ is a time-domain filter algorithm which uses set of mathematical equations and data inputs to estimate the factors such as position, velocity and true values of measurements of an object once the measurement values are uncertainty [1]. This linear quadratic estimation (LQE) is widely used for surveillance, navigation, control of aircraft and spacecraft. The common applications of adapting Kalman filters are object tracking and motion detection in dynamically positioned vehicles [2]. Kalman filter gives system state estimation as mean of the system's predicted state and the new measurement using the weighted values calculated from the measure of the estimated uncertainty of predicted system's state termed as covariance [3]. Since, Kalman filters are recursive in nature, these processes are reiterated at each time step which results in a new estimate and updated covariance with predicted measurements established in the subsequent iterations. For non-

Praveenkumar Babu and Eswaran Parthasarathy are with the Department of Electronics and Communication Engineering, SRM Institute of Science and Technology, Kattankulathur, TN 603203 India (e-mail: praveenb2@srmist.edu.in) (e-mail: eswaranp@srmist.edu.in). linear systems, Extended Kalman filters (EKF) and Unscented Kalman filters (UKF) can be applied [4], [5].

The proposed multi-dimensional Kalman filter provides the process estimation by means of feedback based on noisy measurements. In this robust algorithm, prediction and correction equations are the two update equations resulting in estimated state process. Prediction or extrapolation equations result in the propagation of estimated present state and correction or update equations are responsible for the introduction of new measurements with the estimation of a posteriori state $\hat{x}_{n \mid n+1}$ and updating error covariance. Using such statistical information, the Kalman filter can estimate the state of dynamic systems with particular types of random behaviour.

Several investigations have been carried out on Kalman filters based linear and non-linear state constraints [6]-[12]. Zhou et al. proposed an indirect prediction technique based on the Adaptive Search Pattern (ASP), which increases the accuracy and robustness of tracking targets [13]. Kalman filter based multiple object tracking methods to achieve robustness [14] and efficient tracking are proposed over the years [15], [16]. Although various criteria for Kalman filter design and modifications to achieve robustness, better tracking accuracy and real-time capability have been proposed and investigated, the relationship between model parameters and performance measures such as process noise covariance and error covariance has not been adapted in recent studies [17]. The major contribution of this paper are as follows:

- A multi-dimensional Kalman filter is proposed for state estimation.

- Analysis and performance of multi-dimensional Kalman filter for path tracking is measured and compared with state-of-the-art Kalman filter.

- The optimization in filter design strategy is analyzed using state estimation values.

Table I gives the description of notations quoted in this work. The remainder of this paper is structured as follows. The analysis of multi-dimensional Kalman filter and its properties are formulated in Section II. The simulation results are discussed in Section III followed with concluding remarks and future work.

\section{Formulation OF MUlti-Dimensional Kalman FILTER}

The proposed multi-dimensional Kalman filter for state estimation thereby reducing noise in the observation signal 
TABLE I: Description of variables

\begin{tabular}{|l|l|}
\hline Notation & Description \\
\hline$\hat{\mathrm{x}}_{n \mid n}$ & State vector for estimation \\
$\hat{\mathrm{x}}_{n \mid n+1}$ & Predicted state vector for $(\mathrm{n}+1)$ step \\
$\hat{\mathrm{u}}_{n \mid n}$ & Deterministic variable \\
$\omega$ & Process Noise vector \\
$F$ & State Transition matrix \\
$G$ & Control matrix \\
$\mathrm{P}_{n \mid n}$ & Estimation of uncertainty for present state \\
$\mathrm{P}_{n \mid n}^{+}$ & Estimation of uncertainty for next state \\
$\mathrm{R}_{n}$ & Measurement uncertainty matrix \\
$Q$ & Process noise matrix \\
$K$ & Kalman gain \\
$H$ & Observation matrix \\
$\hat{\mathrm{x}}_{n \mid n-1}$ & Predicted state vector for (n-1) step \\
$\mathrm{Z}_{n}$ & Measurement state vector \\
$A$ & Dynamics System matrices \\
$B$ & Input matrix \\
$C$ & Output matrix \\
$D$ & Feedback or Input matrices \\
$n$ & Discrete Time Index \\
\hline
\end{tabular}

is shown in Figure 1. There are several steps involved in the process of estimating measurement values and uncertainties. The initialization is performed only once to estimate initial system state $x_{(0 \mid 0)}$ and initial state uncertainty $P_{(0 \mid 0)}$. It is followed by prediction. It involves in the measurement process which provides $Z_{n}$ and $R_{n}$. The present system state can be estimated by state update process. Kalman gain $\left(K_{n}\right)$ can be calculated based on these inputs. The prediction or model forecast process estimates the present system state and its uncertainty to the next system state, based on the dynamic model of the system. In this section, the equations of multidimensional Kalman filters are derived in the matrix form because the implementation of Kalman Filter in matrix equations reduces calculation time [18]. For example, the state vector that describes the airplane position, velocity and acceleration is nine-dimensional which is given by,

$$
\left\{\begin{array}{l}
x_{n}=x_{n-1}+x_{n-1}^{\star} \delta t+0.5 x_{n-1}^{\star \star} \delta t^{2} \\
y_{n}=y_{n-1}+y_{n-1}^{\star} \delta t+0.5 y_{n-1}^{\star \star} \delta t^{2} \\
z_{n}=z_{n-1}+z_{n-1}^{\star} \delta t+0.5 z_{n-1}^{\star \star} \delta t^{2} \\
x_{n}^{\star}=x_{n-1}^{\star} t+x_{n-1}^{\star \star} \delta t \\
y_{n}^{\star}=y_{n-1}^{\star} t+y_{n-1}^{\star \star} \delta t \\
z_{n}^{\star}=z_{n-1}^{\star} t+z_{n-1}^{\star \star} \delta t \\
x_{n}^{\star \star}=x_{n-1}^{\star \star} \\
y_{n}^{\star \star}=x_{n-1}^{\star \star} \\
z_{n}^{\star \star}=x_{n-1}^{\star \star}
\end{array}\right.
$$

The properties of multi-dimensional Kalman filter are as follows.

- The stability of Kalman filter is asymptotic such that the solution will become idle to its initial conditions provided that the values of process noise covariance matrix $\left(\left(Q_{n}\right)\right)$ and measurement noise covariance $\left(R_{n}\right)$ are bounded.

- The system is completely controllable and observable if and only if $P_{00}$.

- The factor filter divergence exists when the system is stable for low error variance.

- The filter gives optimal solutions for Gaussian sequences only.

- While it is feasible to estimate the $\left(\left(R_{n}\right)\right)$ and $\left(\left(Q_{n}\right)\right)$ must be constructed in order to adapt various conditions.

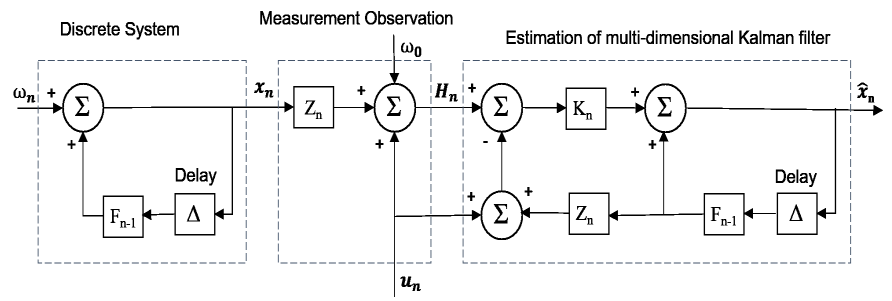

Fig. 1: Structure of proposed multi-dimensional Kalman filter

\section{A. Prediction (or) forecast model}

Equation (1) characterize a stochastic time-variant linear system which can extrapolate state vector from the present state (n) to the next step $(n+1)$. Based on the state extrapolation equation, the future system state can be predicted based on the information of the present state. Consider a discrete-time linear stochastic state space system is given by,

$$
\hat{x}_{n}^{+}=F \hat{x}_{n \mid n}+G \hat{u}_{n \mid n}+\omega_{n} .
$$

The measurement equation gives the true state of the system with observation matrix $(\mathrm{H})$ is given by,

$$
z_{n}=H_{n} x_{n}+v_{n}
$$

The mean and error covariance of the initial state are:

$$
\begin{gathered}
E\left(x_{0}\right)=\mu_{0} . \\
P_{(0 \mid 0)}=E\left[\left(x_{n}-\mu_{x}\right)\left(x_{n}-\mu_{x}\right)^{T}\right] .
\end{gathered}
$$

The predicted state equation is given by,

$$
\begin{gathered}
\hat{x}_{n \mid n}^{+}=E\left[x_{n}\right]=E\left[F \hat{x}_{n \mid n}+G \hat{u}_{n \mid n}+\omega_{n}\right] . \\
\hat{x}_{n \mid n}^{+}=E\left[F \hat{x}_{n \mid n}+G \hat{u}_{n \mid n}\right] . \\
\hat{x}_{n \mid n}^{+}=F \hat{x}_{n \mid n}+G \hat{u}_{n \mid n} .
\end{gathered}
$$

The predicted error of state equation is:

$$
\begin{gathered}
e_{n}=x_{n}-\hat{x}_{n \mid n}=F\left(\hat{x}_{n}-\hat{x}_{n \mid n}^{-}\right)+\omega_{n} . \\
e_{n}=F\left(\hat{e}_{n \mid n}\right)+\omega_{n} .
\end{gathered}
$$

From (4) and (7), we get the covariance equation for next predicted state and is represented as,

$$
\hat{P}_{n \mid n}^{+}=F \hat{P}_{n \mid n} F^{T}+Q .
$$

where $\mathrm{Q}=\left[\begin{array}{cccc}q_{1} & 0 & \ldots & 0 \\ 0 & q_{1} & \ldots & 0 \\ \vdots & \vdots & \ddots & \vdots \\ 0 & 0 & \ldots & q_{n}\end{array}\right]$

The State Update Equation in the matrix form is expressed by,

$$
\hat{x}_{n \mid n}=\hat{x}_{n \mid n}^{-}+K_{n}\left(Z_{n}-H \hat{x}_{n \mid n}^{-}\right) .
$$




\section{B. Correction (or) Data Assimilation}

The present state estimate uncertainty is measured $P_{(n \mid n)}$ using Kalman gain $\left(K_{n}\right)$ which gives the actual measurements and present state estimate. The covariance update equation to measure uncertainty is given by,

$$
\begin{gathered}
P_{n \mid n}=E\left[\left(\left(I-K_{n} H\right)\left(x_{n}-\hat{x}_{n \mid n}\right)-K_{n} \nu_{n}\right)\right. \\
\left.\times\left(\left(I-K_{n} H\right)\left(\left(x_{n}-\hat{x}_{n \mid n}\right)-K_{n} \nu_{n}\right)\right)^{T}\right], \\
\left.P_{n \mid n}=\left(I-K_{n} H\right) P_{n \mid n}^{-}\right)\left(I-K_{n} H\right)^{T} \\
\quad+K_{n} R_{n} K_{n}^{T} .
\end{gathered}
$$

The Kalman gain in matrix notation is expressed as,

$$
K_{n}=P_{n \mid n}^{-} H^{T}\left(H P_{n \mid n}^{-} H^{T}+R_{n}\right)^{-1} .
$$

The estimation of Kalman gain is important because the smallest error in computing can lead to huge computation errors. The subtraction $\left(I-K_{n} H\right)$ can lead to non-symmetric matrices results due to the floating-point errors. Hence, this equation is numerically unstable. In order to get the simplified form of covariance update equation, substitute (13) in (12), we get

$$
\begin{gathered}
P_{n \mid n}=\left[P_{n \mid n}^{-}-\left(P_{n \mid n}^{-} K_{n} H-P_{n \mid n}^{-} K_{n}^{T} H^{T}\right)\right] \\
+K_{n}\left(H P_{n \mid n}^{-} H_{R n}^{T}\right) K_{n}^{T}, \\
P_{n \mid n}=\left[P_{n \mid n}^{-}-\left(P_{n \mid n}^{-} K_{n}^{T} H^{T}\right)\right] \\
-K_{n} H P_{n \mid n}^{-}+P_{n \mid n}^{-} K_{n}^{T} H^{T}, \\
P_{n \mid n}=P_{n \mid n}^{-}-K_{n} H P_{n \mid n} . \\
P_{n \mid n}=\left(I-K_{n} H\right) P_{n \mid n}^{-} .
\end{gathered}
$$

Equation (18) gives the simplified form of updated covariance equation. Figure 2 gives the detailed description of parameters used in Kalman filtering algorithm for tracking application. The steps involved in multi-dimensional Kalman filtering algorithm are follows,

- INITIALIZE the system state $\left(\hat{x}_{n \mid n}\right)$ and state uncertainty $\left(P_{n \mid n}\right)$.

- MEASURE the system state $\left(Z_{n}\right)$ and uncertainty $\left(R_{n}\right)$.

- ESTIMATE the state equation $\left(\hat{x}_{n}^{+}=F \hat{x}_{n \mid n}+G \hat{u}_{n \mid n}+\right.$ $\left.\omega_{n}\right)$.

- CAlCUlate the covariance extrapolation $\left(P_{n \mid n}^{+}=F P_{n \mid n} F^{T}+Q\right)$.

- CALCUlate Kalman $\left(K_{n}=P_{n \mid n}^{-} H^{T}\left(H P_{n \mid n}^{-} H^{T}+R_{n}\right)^{-1}\right)$.

- UPDATE state $\left(\hat{x}_{n \mid n}=\hat{x}_{n \mid n}^{-}+K_{n}\left(Z_{n}-H \hat{x}_{n \mid n}^{-}\right)\right) \quad$ and covariance equation,

$\left(P_{n \mid n}=\left(I-K_{n} H\right) P_{n \mid n}^{-}\left(I-K_{n} H\right)^{T}+K_{n} R_{n} K_{n}^{T}\right)$.

- PREDICT the extrapolation from present state to next state.

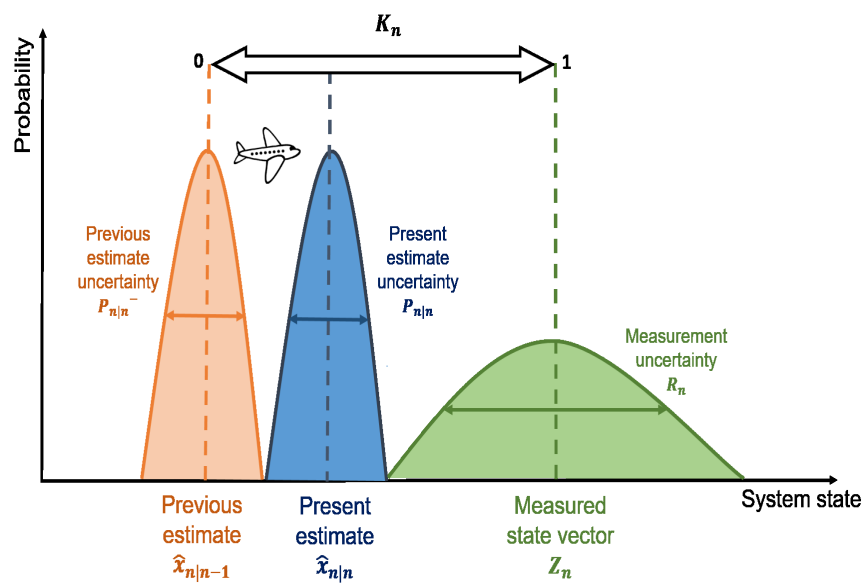

Fig. 2: Illustration of multi-dimensional Kalman filter parameters for aircraft tracking application.

\section{RESUlTS AND DiscUSSION}

The analysis of path tracking measurements with measured, actual and estimated values are shown in Figure 3. In multi-dimensional Kalman filter, error covariance $\left(P_{n \mid n}\right)$ and Kalman gain $\left(K_{n}\right)$ are updated with time and are expressed in Equations (18) and (14) respectively. It is observed that the estimated values based on these updated equations produce effective path tracking results.
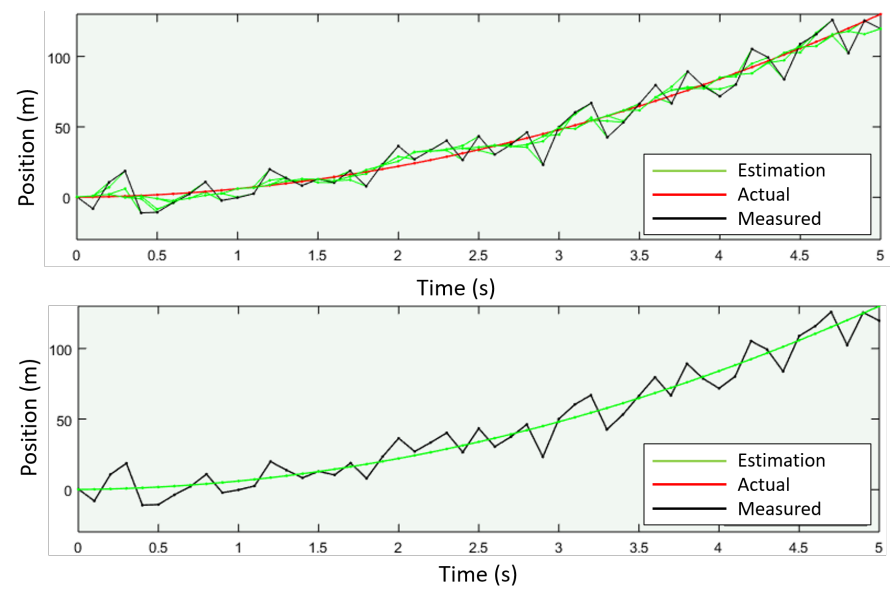

Fig. 3: Analysis of path tracking compared with Kalman filter (top) and multi-dimensional Kalman filter (bottom).

The multi-dimensional Kalman filtering algorithm estimates the output covariance during the estimation. Figure 4 gives the output covariance which tends to produce steady-state values after four number of samples. Since then, the proposed filter response achieves same performance as the steady-state response. By means of the steady-state instance, the error due to measurement noise is reduced by the filter responses. The computation of the error covariance after filtering (estimation error covariance) and before filtering (measurement error covariance) can be done for minimization of noise measurements. 




Fig. 4: Output covariance plot reaching steady state after four number of samples.

Comparison of true responses and filtered responses for given number of samples is measured and is shown in Figure 5. The experimental results shown that filtered responses is very much similar to true responses of produced by multidimensional Kalman filter.
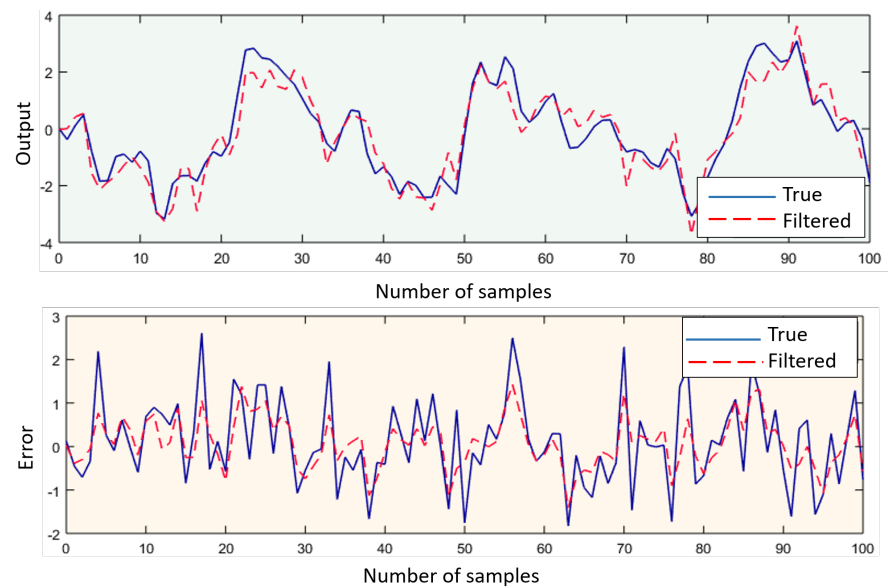

Fig. 5: True response vs Filtered response using proposed multi-dimesional Kalman filter

\section{CONCLUSION}

This paper proposed multi-dimensional Kalman filter for tracking applications. The proposed is analyzed based on the steady-state process estimation for linear dynamic systems. Based on filtering algorithm, state vector $\left(\hat{x}_{n \mid n}\right)$ and covariance equations $\left(P_{n \mid n}\right)$ are updated in order to achieve high Kalman gain $\left(K_{n}\right)$. From the simulation results, we identified that the conditions under which the proposed multidimensional filter outperforms the conventional Kalman filter for different number of samples in terms of tracking accuracy.

The foremost future objective is to implement the multidimensional Kalman filter on FPGA reconfigurable logic to meet parallelism and flexibility.
APPENDIX

$$
\begin{gathered}
E\left[\omega_{n}\right]=E\left[\nu_{n}\right]=0 ; \hat{x}_{n \mid n}=E\left[x_{n} \mid Z_{n}\right] \\
E\left[\omega_{n} \omega_{n}^{T}\right]=Q ; E\left[\nu_{n} \nu_{n}^{T}\right]=R
\end{gathered}
$$

Derivation of (4), The covariance matrix of the vector is given by,

$$
P_{n \mid n}=E\left[\left(x_{n}-\mu_{x}\right)\left(x_{n}-\mu_{x}\right)^{T}\right]
$$

Proof: From the above equation (21), we get

$$
\begin{array}{r}
\mathbf{P}_{n \mid n}=E\left[\begin{array}{cccc}
\left(x_{1}-\mu_{x_{1}}\right)^{2} & \left(x_{1}-\mu_{x_{1}}\right)\left(x_{2}-\mu_{x_{2}}\right) & \cdots & \left(x_{1}-\mu_{x_{1}}\right)\left(x_{n}-\mu_{x_{n}}\right) \\
\left(x_{2}-\mu_{x_{2}}\right)\left(x_{1}-\mu_{x_{1}}\right) & \left(x_{2}-\mu_{x_{2}}\right)^{2} & \cdots & \left(x_{2}-\mu_{x_{2}}\right)\left(x_{n}-\mu_{x_{n}}\right) \\
\vdots & \vdots & \ddots & \vdots \\
\left(x_{k}-\mu_{x_{k}}\right)\left(x_{1}-\mu_{x_{1}}\right) & \left(x_{k}-\mu_{x_{k}}\right)^{2} & \cdots & \left(x_{n}-\mu_{x_{n}}\right)^{2}
\end{array}\right] \\
\mathbf{P}_{n \mid n}=E\left(\left[\begin{array}{c}
\left(x_{1}-\mu_{x_{1}}\right) \\
\left(x_{2}-\mu_{x_{2}}\right) \\
\vdots \\
\left(x_{k}-\mu_{x_{k}}\right)
\end{array}\right]\left[\begin{array}{cccc}
{\left[\left(x_{1}-\mu_{x_{1}}\right)\right.} & \left(x_{2}-\mu_{x_{2}}\right) & \cdots & \left.\left(x_{k}-\mu_{x_{k}}\right)\right]
\end{array}\right)\right.
\end{array}
$$

Hence, we get

$P_{n \mid n}=E\left[\left(x_{n}-\mu_{x}\right)\left(x_{n}-\mu_{x}\right)^{T}\right]$.

Derivation of (11), We know that,

$$
\begin{gathered}
\hat{x}_{n \mid n}=E\left[\left(x_{n} \mid Z_{n}\right)\right]+E\left[\left(x_{n} \mid Z_{n-1}\right]\right. \\
\hat{x}_{n \mid n}=\hat{x}_{n \mid n}^{-}+K_{n}\left(Z_{n}-H \hat{x}_{n \mid n}^{-}\right) \\
\hat{x}_{n \mid n}=\left(I-K_{n} H\right) \hat{x}_{n \mid n}^{+}+K_{n} Z_{n}
\end{gathered}
$$

Derivation of (12) and (13): From (4) and (8), we get

$$
\begin{aligned}
P_{n \mid n} & =E\left[\left(\left(I-K_{n} H\right)\left(x_{n}-\hat{x}_{n \mid n}\right)-K_{n} \nu_{n}\right)\right. \\
& \left.\times\left(\left(I-K_{n} H\right)^{T}\left(\left(x_{n}-\hat{x}_{n \mid n}\right)^{T}-K_{n} \nu_{n}\right)\right)^{T}\right],
\end{aligned}
$$

We know that $(A B)^{T}=B^{T} A^{T}$,

$$
\begin{aligned}
P_{n \mid n} & =E\left[\left(\left(I-K_{n} H\right)\left(x_{n}-\hat{x}_{n \mid n}\right)^{T}-\left(I-K_{n} H\right)^{T}\right.\right. \\
& -\left(( I - K _ { n } H ) \left(\left(x_{n}-\hat{x}_{n \mid n}\right)\left(K_{n} \nu_{n}\right)^{T}\right.\right. \\
& \left.-\left(K_{n} \nu_{n}\right)\left(x_{n}-\hat{x}_{n \mid n}\right)^{T}-\left(I-K_{n} H\right)^{T}+\left(K_{n} \nu_{n}\right)^{T}\right],
\end{aligned}
$$

Apply $E(X+Y)=E(X)+E(Y)$ and $E\left[\left(x_{n}-\hat{x}_{n \mid n}\right)\right]=0$,

$$
\begin{aligned}
& P_{n \mid n}=E\left[\left(I-K_{n} H\right)\left(x_{n}-\hat{x}_{n \mid n}\right)\left(x_{n}-\hat{x}_{n \mid n}\right)^{T}\right. \\
& \left.\quad-\left(I-K_{n} H\right)^{T}\right]+E\left[\left(K_{n} \nu_{n} K_{n}^{T} \nu_{n}^{T}\right)\right],
\end{aligned}
$$

Apply Expectation property, $E(a X)=a E(X)$ in (27),

$$
\begin{aligned}
P_{n \mid n} & \left.=\left(I-K_{n} H\right) P_{n \mid n}^{-}\right)\left(I-K_{n} H\right)^{T} \\
& +K_{n} R_{n} K_{n}^{T} .
\end{aligned}
$$

Derivation of (14): Equation (28) can be written as,

$$
\begin{aligned}
P_{n \mid n} & =\left(P_{n \mid n}^{-}-P_{n \mid n}^{-} K_{n} H\right)\left(I-K_{n}^{T} H^{T}\right) \\
& +K_{n} R_{n} K_{n}^{T} .
\end{aligned}
$$


Apply trace property, we get

$$
\begin{aligned}
\operatorname{tr}\left(P_{n \mid n}\right) & =\operatorname{tr}\left[\left(P_{n \mid n}^{-}-P_{n \mid n}^{-} K_{n} H\right)\left(I-K_{n}^{T} H^{T}\right)\right. \\
& \left.+K_{n} R_{n} K_{n}^{T}\right] .
\end{aligned}
$$

Differentiate trace of $P(n \mid n)^{-}$with respect to $K_{n}$, then (30) becomes,

$$
\begin{aligned}
& \frac{d \operatorname{tr}\left(P_{n \mid n}\right)}{d K_{n}}=0-2\left(H P_{n \mid n}^{-}\right)^{T} \\
& +2 K_{n}\left(H P_{n \mid n}^{-} H^{T}+R_{n}\right)=0 \\
& \left(H P_{n \mid n}^{-}\right)^{T}=K_{n}\left(H P_{n \mid n}^{-} H^{T}+R_{n}\right) \\
& K_{n}=\left(H P_{n \mid n}^{-}\right)^{T}\left(H P_{n \mid n}^{-} H^{T}+R_{n}\right)^{-1}
\end{aligned}
$$

Apply matrix property $(A B)^{T}=B^{T} A^{T}$ in (33), we get gain equation for proposed multi-dimensional Kalman filter.

$$
K_{n}=P_{n \mid n}^{-} H^{T}\left(H P_{n \mid n}^{-} H^{T}+R_{n}\right)^{-1} .
$$

\section{ACKNOWLEDGMENT}

We thank SRM Institute of Science and Technology for the support to carry out this research work.

\section{REFERENCES}

[1] R. E. Kalman, "A new approach to linear filtering and prediction problems," Journal of Fluids Engineering, vol. 82, no. 1, pp. 35-45, 1960.

[2] Q. Li, R. Li, K. Ji and W. Dai, "Kalman Filter and Its Application," in Proc. $8^{\text {th }}$ International Conference on Intelligent Networks and Intelligent Systems (ICINIS), China, 2015, pp. 74-77.

[3] F. A. Faruqi and R. C. Davis, "Kalman filter design for target tracking," IEEE Transactions on Aerospace and Electronic Systems, vol. AES-16, no. 4, pp. 500-508, July 1980, 10.1109/TAES.1980.308980.

[4] Simon J. Julier and Jeffrey K. Uhlmann, "New extension of the Kalman filter to non-linear systems," in Proc. SPIE 3068, Signal Processing, Sensor Fusion, and Target Recognition VI, 1997.

[5] Peng Gu, Zhongliang Jing and Liangbin Wu, "Adaptive fading factor unscented Kalman filter with application to target tracking," Aerospace Systems, vol. 4, pp. 1-6, 2021.

[6] K. Mahata and T. Söderström, "Improved estimation performance using known linear constraints," Automatica, vol. 40, no. 8, pp. 1307-1318, 2004, 10.1016/j.automatica.2004.03.001.

[7] S. Ko and R. Bitmead, "State estimation for linear systems with state equality constraints," Automatica, vol. 43, no. 8, pp. 1363-1368, 2007.

[8] D. Simon, "Kalman filtering with state constraints: a survey of linear and nonlinear algorithm," IET control theory and application, vol. 4, no. 8, pp. 1303 - 1318, 2010.

[9] C. Campestrini et al. , "A comparative study and review of different Kalman filters by applying an enhanced validation method," Journal of Energy Storage, vol. 8, pp. 142-159, 2016.

[10] X. Fu, Y. Jia, J. Du and F. Yu, "Kalman filtering with multiple nonlinear-linear mixing state constraints," in Proc. $49^{\text {th }}$ IEEE Conference on Decision and Control (CDC), China, 2010, pp. 340-345.

[11] A. Umamageswari , J. Joseph Ignatious , R. Vinodha, "A comparative study of Kalman Filter, extended Kalman Filter and unscented Kalman Filter for Harmonic Analysis of the non-stationary signals," International Journal of Scientific Engineering Research, vol. 3, no. 7, pp. 1-9, 2012.

[12] W. Wang, C. K. Tse and S. Wang, "Dynamic State Estimation of Power Systems by p -Norm Nonlinear Kalman Filter,' IEEE Transactions on Circuits and Systems I: Regular Papers, vol. 67, no. 5, pp. 1715-1728, May 2020, 10.1109/TCSI.2020.2965141.

[13] W. Zhou and J. Hou, "A new adaptive high-Order Unscented Kalman Filter for improving the accuracy and robustness of target tracking," IEEE Access, vol. 7, pp. 118484-118497, 2019.

[14] Xiao Lu, Lihua Xie, Huanshui Zhang, and Wei Wang, "Robust Kalman Filtering for Discrete-Time Systems With Measurement Delay," IEEE Transactions on Circuits and Systems II: Express Briefs, vol. 54, no. 6 pp. 522-526, 2007, 10.1109/TCSII.2007.892223.

[15] X. Li, K. Wang, W. Wang and Y. Li, "A multiple object tracking method using Kalman filter," in Proc. IEEE International Conference on Information and Automation, Harbin, China, 2010, pp. 1862-1866, 10.1109/ICINFA.2010.5512258.

[16] Y. Chen, P. Chen and S. Chien, "An objective tracking method based on Kalman filter," in Proc. International Conference on Advanced Robotics and Intelligent Systems (ARIS), Taipei, Taiwan, 2016, pp. 1-1, 10.1109/ARIS.2016.7886626.

[17] K. Saho and M. Masugi, "Performance analysis of $\alpha-\beta$ $\gamma$ tracking filters using position and velocity measurements," EURASIP J. Adv. Signal Process. 35, pp. 1-15, 2015, 10.1186/s13634-015-0220-3.

[18] A. Mills, P. H. Jones and J. Zambreno, "Parameterizable FPGA-Based Kalman Filter Coprocessor Using Piecewise Affine Modeling," in Proc. IEEE International Parallel and Distributed Processing Symposium Workshops (IPDPSW), Chicago, IL, USA, 2016, pp. 139-147. 